\title{
AVALIAÇÃO DA CARTILAGEM DO JOELHO PELA RESSONÂNCIA MAGNÉTICA
}

\author{
MRI EVALUATION OF KNEE CARTILAGE
}

Marcelo Bordalo Rodrigues' ${ }^{1}$, Gilberto Luís Camanho²

\section{RESUMO}

A ressonância magnética ( $\mathrm{RM})$, através de sua capacidade de caracterizar partes moles de forma não invasiva, tornou-se excelente método na avaliação da cartilagem. O desenvolvimento de novos e mais rápidos métodos possibilitaram aumento da resolução e do contraste na avaliação da estrutura condral, com maior precisão diagnóstica. Além disso, foram desenvolvidas técnicas fisiológicas de avaliação da cartilagem, capazes de detectar alterações precoces, antes do aparecimento das fissuras e erosões. Neste artigo de atualização serão discutidas e demonstradas as diversas técnicas de avaliação condral pela RM no joelho.

Descritores - Traumatismos do joelho; Imagem por ressonância magnética; Cartilagem articular; Articulação do joelho

\section{ABSTRACT}

Magnetic resonance imaging (MRI), through its ability to characterize soft tissue noninvasively, has become an excellent method in the evaluation of cartilage. The development of new and faster methods allowed increased resolution and contrast in the evaluation of chondral structure, with greater diagnostic accuracy. In addition, techniques were developed physiological assessment of cartilage which can detect early changes before the appearance of cracks and erosions. In this updated article will be discussed and demonstrated various techniques for assessing chondral knee by MRI.

Keywords - Knee injuries; Magnetic resonance imaging; Articular cartilage; Knee joint

\section{INTRODUÇÃO}

A cartilagem hialina é um tecido conectivo fino composto de complexa rede de fibras colagenosas, água e proteoglicanos $^{(1)}$. As lesões condrais geralmente progridem lentamente e as manifestações clínicas ocorrem tardiamente no processo.

A radiografia simples permite a avaliação indireta da cartilagem e constitui boa opção na avaliação da doença degenerativa, tendo em vista as atuais opções terapêuticas. O estudo direto da cartilagem hialina através da ressonância magnética é indicado principalmente nos casos precoces de osteoartrose, com pouca ou nenhuma alteração à radiografia simples.

Com o desenvolvimento dos métodos de imagem é possível avaliar o acometimento da cartilagem em fases cada vez mais precoces e de forma cada vez mais precisa. Esta evolução dos métodos de imagem avança em conjunto com o desenvolvimento das novas drogas no tratamento da degeneração condral. Entretanto, os achados de imagem devem ser avaliados com parcimônia e sempre valorizados apenas em conjunto com a sintomatologia do paciente, com intuito de se evitar tratamentos desnecessários.

\section{Ressonância magnética}

A ressonância magnética (RM) permite avaliação direta da cartilagem hialina, refletindo sua complexa bioquímica e histologia. É considerada o melhor método não invasivo para avaliação da cartilagem articular devido ao seu alto contraste de partes moles ${ }^{(2-5)}$. Utilizando técnicas convencionais, fornece informações sobre a espessura condral, alterações morfológicas da superfície condral,

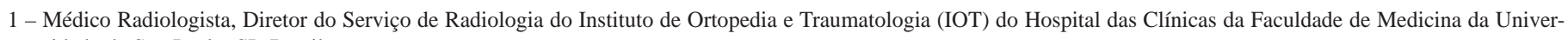
sidade de São Paulo, SP, Brasil.

2 - Professor Titular do Departamento de Ortopedia e Traumatologia da Faculdade de Medicina da Universidade de São Paulo, SP, Brasil 
alterações de sinal intrassubstanciais da cartilagem e também avalia alterações do osso subcondral. Através de técnicas mais recentes, a RM fornece informações sobre as características bioquímicas e fisiológicas da cartilagem hialina. Desta forma, a RM torna-se cada vez mais sensível na detecção das lesões condrais precoces.

\section{Técnicas convencionais}

O estudo da cartilagem hialina depende da obtenção de imagens com alta resolução espacial ${ }^{(6)}$. O desenvolvimento dos equipamentos de RM aumentou consideravelmente durante os últimos anos, com melhorias nos gradientes e nas bobinas de radiofrequência. Com os equipamentos atuais, os magnetos de 1,5 Tesla propiciam imagens de cartilagem com ótima resolução espacial. As sequências de RM utilizadas para estudo da cartilagem articular são as pesadas em T1, densidade de prótons e T2. As sequências ponderadas em T1 não são úteis para avaliar a cartilagem e, sim, para avaliar o osso subcondral. As sequências ponderadas em T2 avaliam o osso subcondral e a interface entre a cartilagem e o líquido sinovial, com menor distinção das alterações de sinal intrínsecas da cartilagem hialina. Para visualização direta da cartilagem hialina a sequência ponderada em densidade de prótons fornece maior contraste de sua estrutura e também avalia a interface cartilagem-líquido sinovial e o osso subcondral. Atualmente a sequência ponderada em densidade de prótons é considerada a com maior acurácia na detecção da lesão condral ${ }^{(7-9)}$. Sequências volumétricas utilizando ecos de gradiente (gradiente eco e SPGR) fornecem imagens com alta resolução espacial, indicadas para a quantificação da morfologia condral. Suas desvantagens são um menor contraste entre a cartilagem e o líquido sinovial e o alto tempo de aquisição ${ }^{(10-13)}$. Entretanto, várias sequências volumétricas baseadas em ecos de gradientes estão em desenvolvimento, com o intuito de se aumentar o contraste da interface cartilagem - líquido sinovial e diminuir o tempo de aquisição ${ }^{(14,15)}$ (Figura 1, A e B).

Nos últimos dois anos, cresceu a utilização dos magnetos de alto campo na prática clínica, especialmente os magnetos de $3 \operatorname{Tesla}^{(16)}$. Estes magnetos possibilitam a aquisição de imagens morfológicas em resoluções espaciais maiores, impossíveis de serem alcançadas em tempo de aquisição razoável pelos aparelhos de 1,5 Tesla (Figura 2). Apesar disto, ainda faltam estudos na literatura para demonstrar se há aumento da acurácia dos magnetos de 3 Tesla em relação aos de 1,5 Tesla na detecção das lesões condrais.

\section{Cartilagem normal}

Utilizando técnicas de RM com alta resolução espacial e um bom contraste de tecidos moles é possível se observar um padrão trilaminar da cartilagem hialina: 1) lâmina superficial com hipossinal; 2) lâmina intermediária com hipersinal; e 3) lâmina profunda com hipossinal e transição em "paliçada” com a zona intermediária. Este aspecto trilaminar é mais evidente nas cartilagens de maior espessura como as da patela e da tróclea femoral (Figura 3).

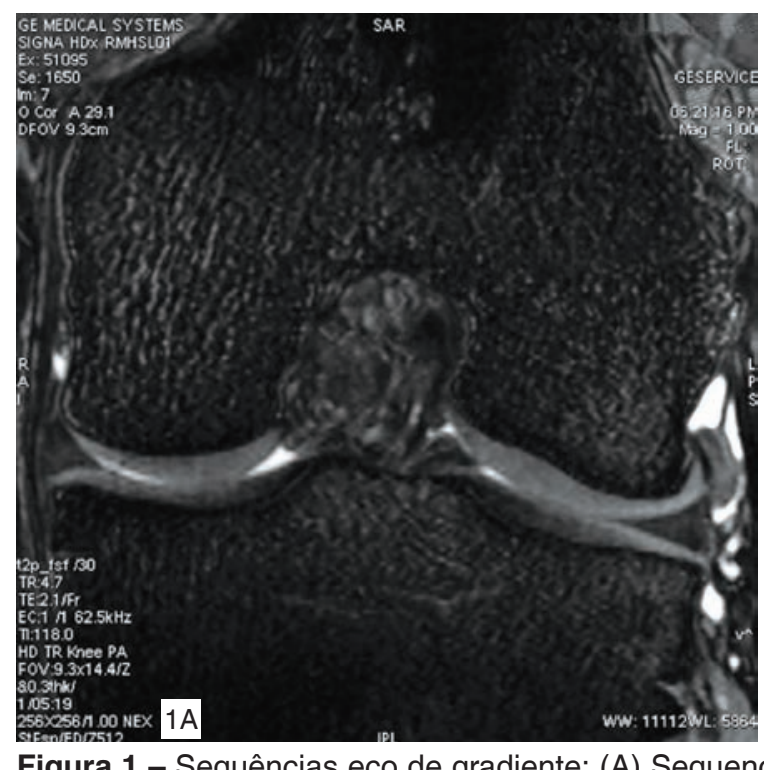

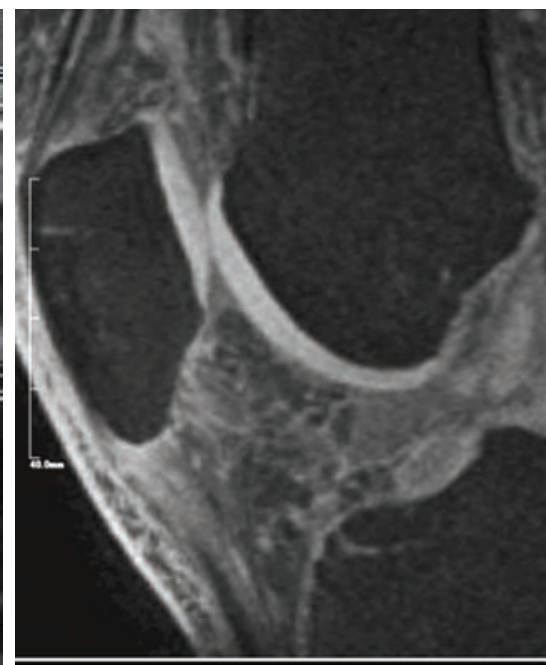

SPGR - T1

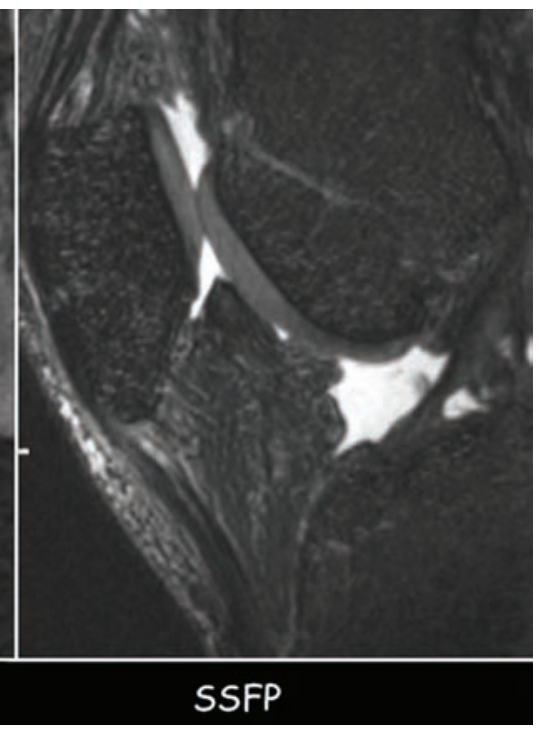

$1 \mathrm{~B}$

Figura 1 - Sequências eco de gradiente: (A) Sequencia SSFP (Steady State Free Precession) no plano coronal. Notar a alta definição da superfície condral dos côndilos femorais e platôs tibiais. (B) Comparação entre as sequências eco de gradiente no mesmo paciente: a sequencia SSFP apresenta uma maior definição de sua superfície e maior contraste com o líquido sinovial em relação a sequencia SPGR (Spoiled Gradient Recalled). 


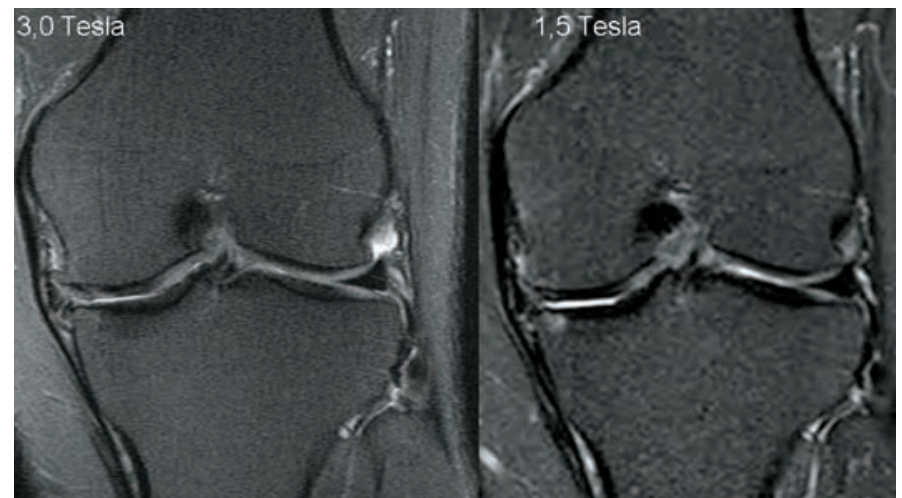

Figura 2 - Comparação entre 3,0 e 1,5 Tesla. Imagens coronais de RM ponderadas em T2 com saturação de gordura do mesmo paciente realizadas em aparelhos de 3,0 e 1,5 Tesla. Existe uma maior resolução espacial na imagem do aparelho 3,0 Tesla, com uma maior definição da espessura condral dos compartimentos medial e lateral. Também há uma maior definição dos contornos dos osteófitos marginais e meniscais.

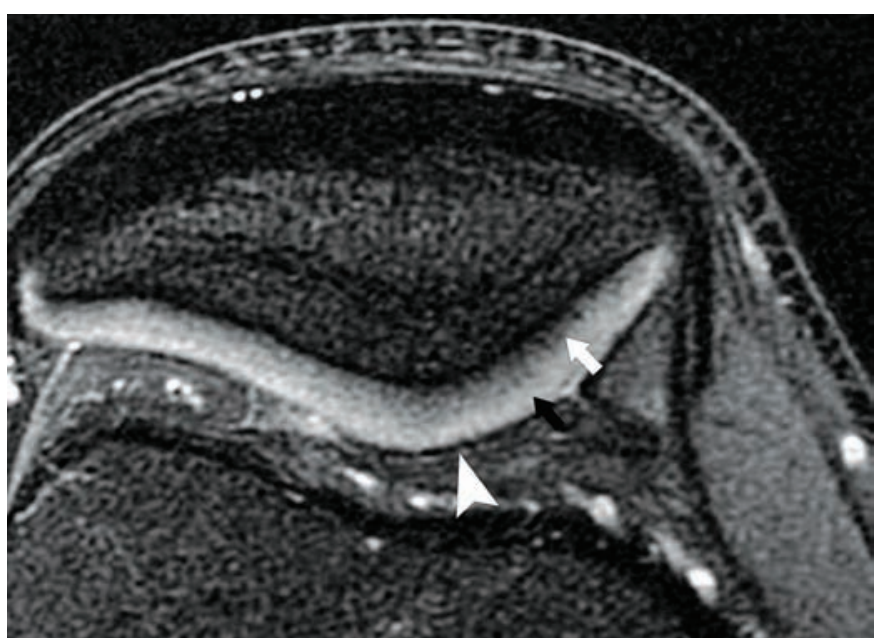

Figura 3 - Aspecto trilaminar da cartilagem hialina. Imagem axial da patela de RM ponderada em T2 com saturação de gordura demonstra o aspecto trilaminar da cartilagem hialina. A camada mais profunda com hipossinal (seta), a camada intermediária com alto sinal (cabeça de seta preta) e a camada superficial com hipossinal (cabeça de seta branca).

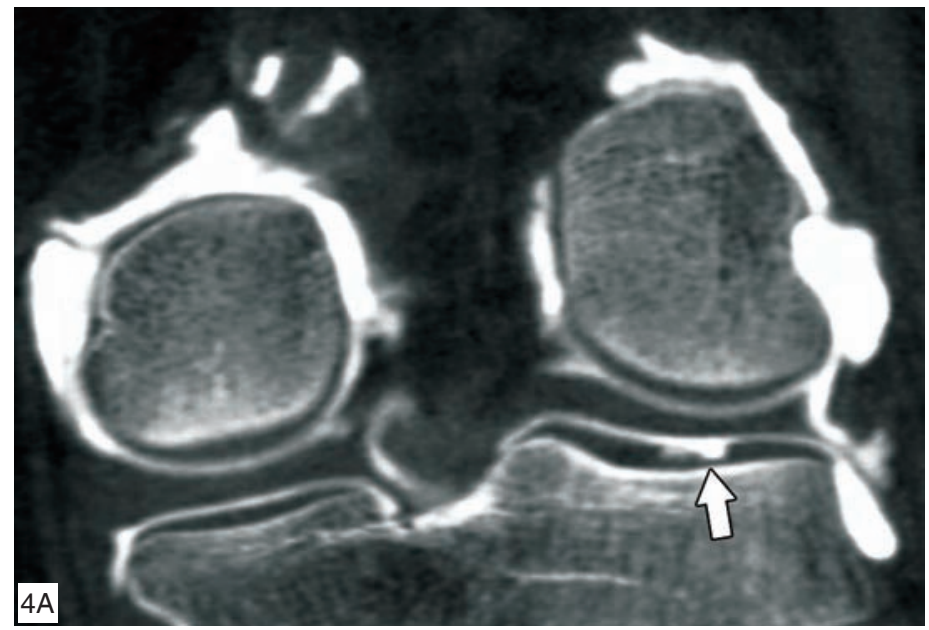

Figura 4 - Locais mais comuns das lesões condrais no joelho. Artro-TC - reformatações coronais. (A) Lesão condral na porção posterior do platôtibial lateral (seta). (B) Lesão condral no aspecto mais interno do côndilo femoral medial (cabeça de seta).

\section{Lesões condrais}

A acurácia da RM na detecção da lesão condral depende da técnica de RM utilizada e, principalmente, do tamanho da lesão. Nas fibrilações superficiais, a RM convencional com protocolos otimizados para avaliação da cartilagem apresenta baixa acurácia diagnóstica. A acurácia é maior nas lesões mais profundas, especialmente naquelas que apresentam mais de $50 \%$ de perda de substância condral, com valores na literatura de 73 a $96 \%{ }^{(17,18)}$.

A lesão condral é diagnosticada por alterações de sinal, espessura e morfologia da cartilagem hialina. O sinal de RM mais específico de lesão condral é a presença de área de elevação do sinal nas sequências ponderadas em DP, T2 e no gradiente eco. Este sinal, porém, não é sensível para determinar lesão. Um estudo demonstrou que $70 \%$ das lesões condrais apresentam alto sinal em relação à cartilagem normal na sequência de densidade de prótons, 20\% apresentam sinal semelhante ao da cartilagem normal (ou seja, lesões não vistas na RM) e 10\% apresentam baixo sinal em relação à cartilagem normal. Também foi demonstrado que as lesões com alto sinal são mais extensas que as lesões com iso ou hipossinal $^{(19)}$.

O afilamento condral, perda na definição dos contornos da cartilagem e irregularidades superficiais são outros sinais que auxiliam na avaliação.

Foi demonstrado que os locais mais frequentes de lesões condrais são o côndilo femoral medial (em seu aspecto mais interno) e o platô tibial lateral (em sua porção mais posterior) ${ }^{(20)}$ (Figura 4, A e B).

Para classificar as lesões condrais pela RM, utilizamos um sistema de classificação baseado nas clas-

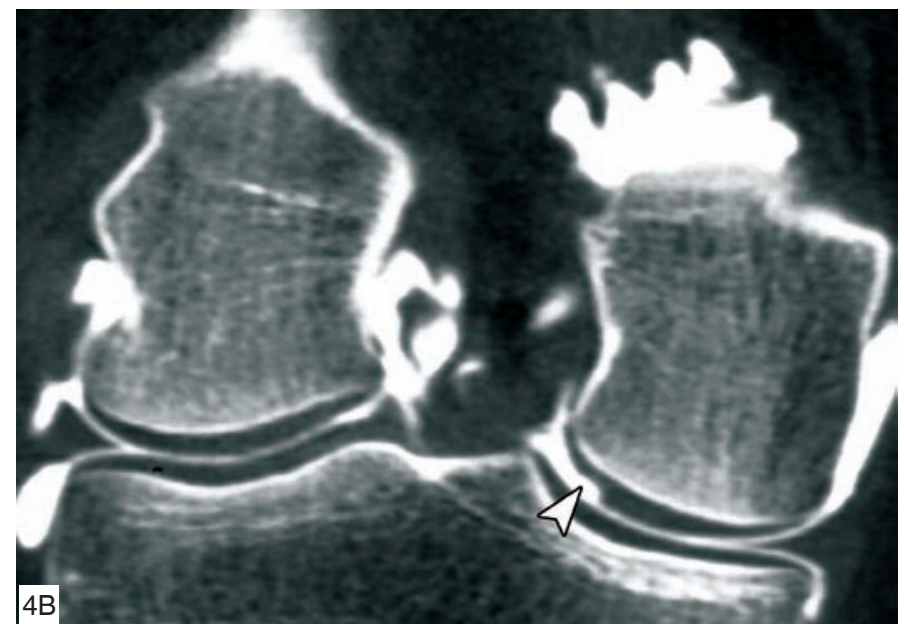


sificações artroscópicas ${ }^{(21-23)}$. A lesão grau I indica alteração de sinal focal intrassubstancial da cartilagem, correspondendo ao amolecimento da cartilagem pela artroscopia. A lesão grau II constitui alteração de sinal superficial, indicando fibrilação ou erosão menor que $50 \%$ da espessura condral. Na lesão grau III, há perda de mais de $50 \%$ da substância condral, podendo haver pequenas áreas atingindo a superfície óssea. A lesão grau IV indica defeito condral extenso de espessura total com edema da medula óssea (Figura 5, A a D).

\section{Alterações do osso subcondral}

A perda da integridade da cartilagem articular pode provocar alterações no osso subjacente como cistos (geodos), esclerose e osteofitose, detectáveis pela RM (Figura 6A a C). Outro achado frequente associado é a elevação de sinal do osso subcondral, denominado edema ósseo que, na realidade, constitui a expressão de várias alterações histológicas como necrose, fibrose e microfraturas trabeculares ${ }^{(24)}$. Foi demonstrado que os cistos subcondrais desenvolvem-se em áreas preexistentes de "edema ósseo subcondral”(25).

\section{Alterações sinoviais}

A lesão condral crônica com destacamento de fragmentos cartilaginosos para a articulação leva à irritação crônica da sinóvia, podendo causar sinovite. Em alguns casos, a resposta sinovial é tão extensa e pode adquirir formas pseudotumorais nos exames por imagem (Figura 7, A a C). Também é comum a presença de corpos livres articulares, especialmente nos recessos suprapatelar, posterior da articulação femorotibial e poplíteo.

\section{Alterações secundárias da articulação}

A artrose também pode levar a algumas alterações secundárias na articulação, entre elas lesão degenerativa do menisco medial e osteonecrose com colapso articular (Figuras 8 e 9, A e B).
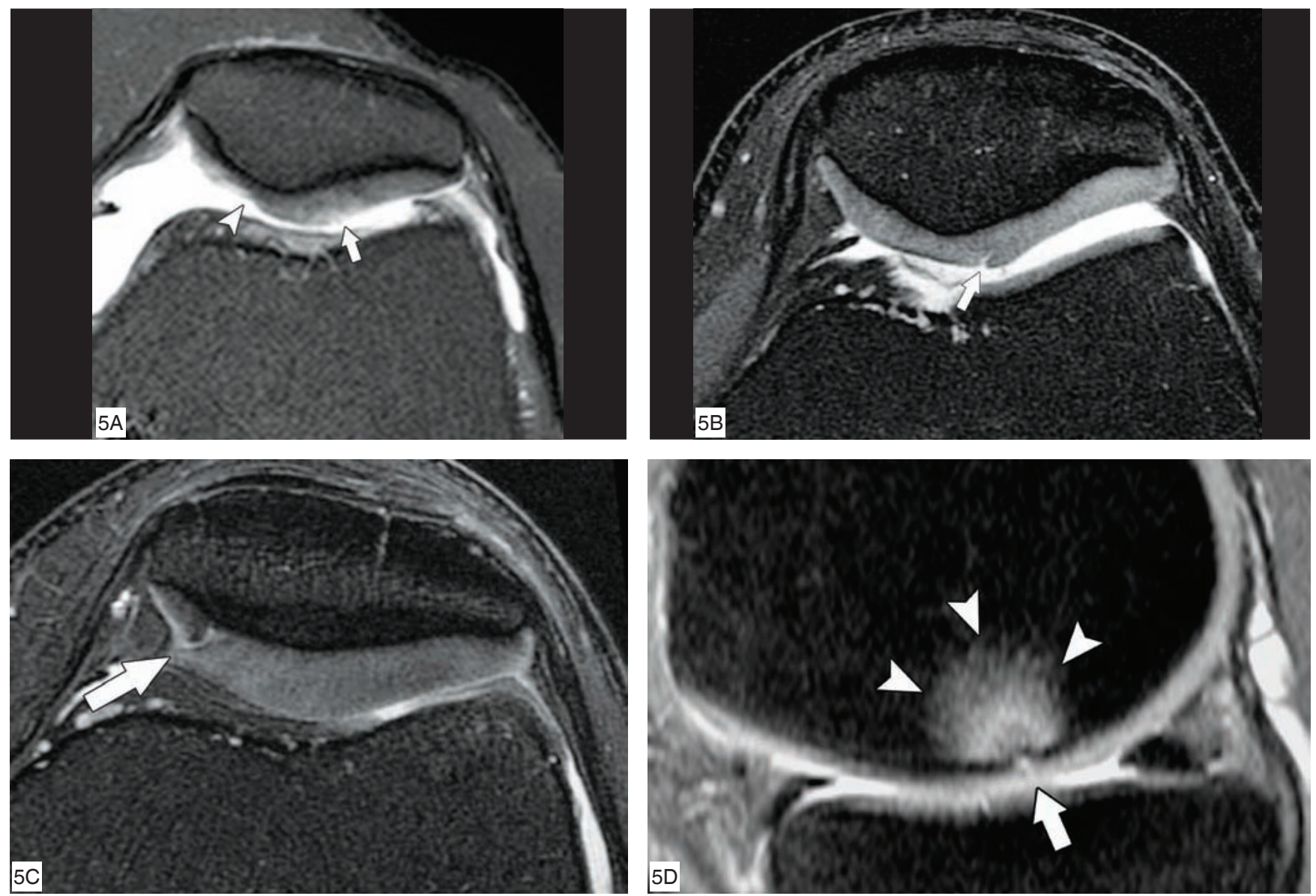

Figura 5 - Classificação das lesões condrais no joelho. Imagens axiais (A a C) e sagital (D) de RM do joelho ponderadas em T2 com saturação de gordura. (A) Tênue elevação do sinal superficial da cartilagem hialina da faceta lateral da patela (seta), indicando condropatia grau I. Também há erosão condral profunda (grau III) na faceta medial (cabeça de seta). (B) Fissura condral acometendo menos de $50 \%$ da espessura total na faceta lateral da patela, indicando condropatia grau II (seta). (C) Fissura condral acometendo mais de $50 \%$ da espessura total da faceta medial da patela, indicando condropatia grau III (seta). (D) Fissura condral profunda no côndilo femoral lateral (seta), atingindo o osso subcondral e com edema da medula óssea adjacente (cabeças de seta), caracterizando condropatia grau IV. 

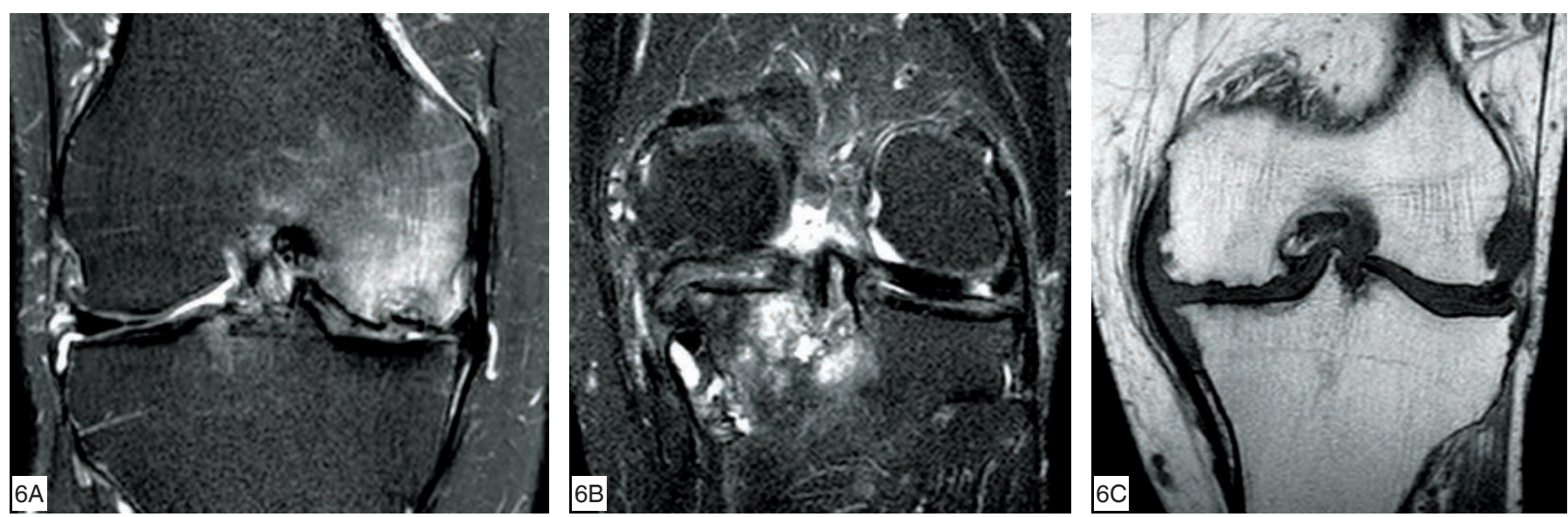

Figura 6 - Alterações subcondrais na RM. Imagens coronais de RM do joelho ponderadas em T2 com saturação de gordura (A e B) e em T1 (C). (A) Redução da espessura condral no côndilo femoral medial, com edema da medula óssea e pequena fratura do osso cortical subcondral. (B) Artropatia degenerativa fêmoro-tibial com cistos subcondrais e edema da medula óssea no platô tibial medial. (C) Artropatia degenerativa fêmoro-tibial medial e lateral com osteófitos marginais.
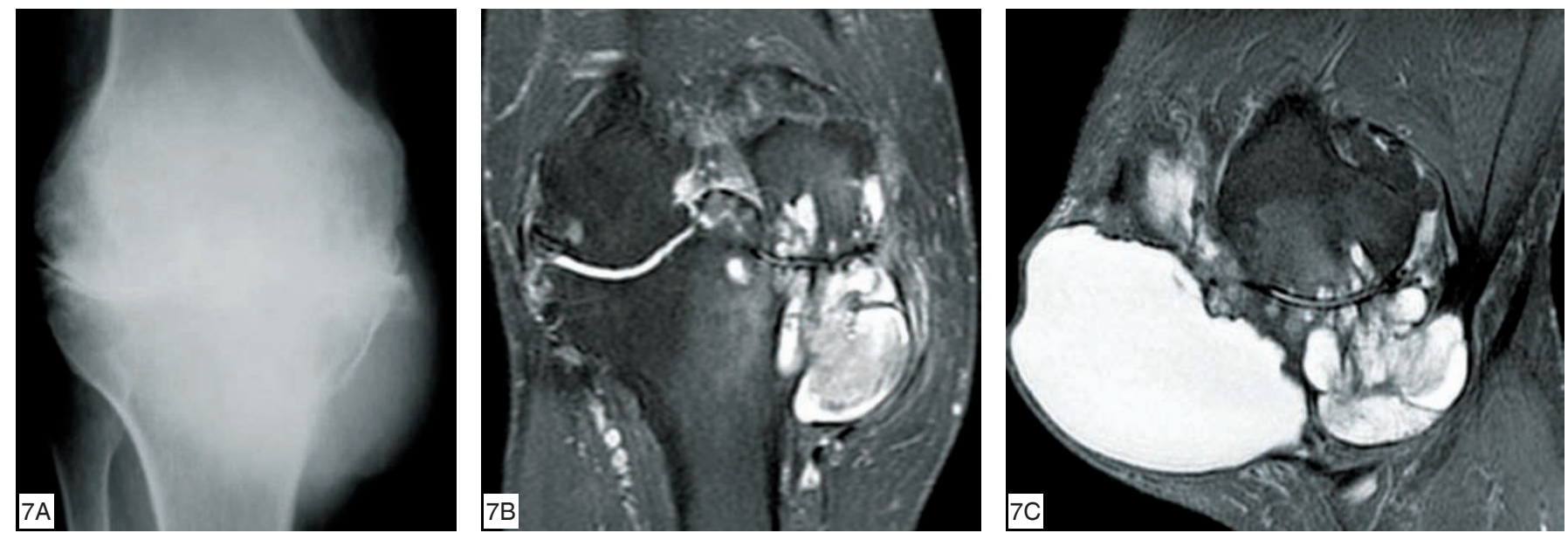

Figura 7 - Metaplasia sinovial secundária a artrose severa com aspecto pseudotumoral. (A) Radiografia AP do joelho demonstra artrose severa grau V de Ahlbäck com aumento de partes moles junto ao platô tibial medial. Imagens coronal (B) e sagital (C) de RM ponderada em T2 com saturação de gordura evidencia grandes cistos subcondrais no platô tibial e côndilo femoral e grande cisto sinovial anterior, secundário a metaplasia.

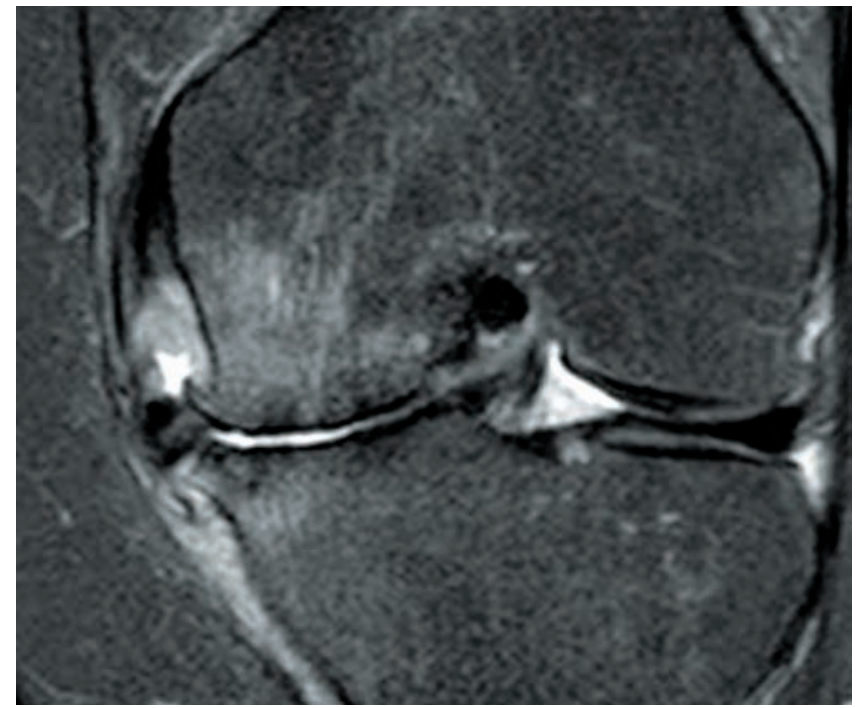

Figura 8 - Lesão degenerativa meniscal secundária a artrose severa. Artropatia degenerativa fêmoro-tibial medial associado a uma extrusão do corpo do menisco medial que apresenta elevação do seu sinal e sinovite ao seu redor, indicando uma lesão degenerativa.

\section{Avaliação fisiológica da cartilagem hialina por ressonância magnética}

A cartilagem hialina é constituída de água (70\% de seu peso), colágeno tipo II e proteoglicanos. Através de técnicas de aquisição e avaliação de imagens por RM é possível avaliar a composição bioquímica da cartilagem hialina. Estas técnicas ainda estão em fase de pesquisa, mas são promissoras em relação à sua aplicação clínica em futuro próximo, especialmente em relação à deteç̧ão precoce da lesão condral, antes de uma lesão macroscópica e anatômica. A seguir serão descritas sucintamente algumas destas técnicas:

- Mapa T2: avalia o conteúdo de água e a ultraestrutura do colágeno do tecido. A medida do tempo de relaxamento T2 demonstra áreas de maior ou menor conteúdo de água, dependendo da lesão condral ${ }^{(26-28)}$. Esta mensuração é representada por um mapa em escala de cores. Aumento 
do tempo de relaxamento T2 em área focal na cartilagem é associada a dano na matriz condral, especialmente perda da integridade do colágeno (Figura 10, A e B).
- Mapa de T1-rho: técnica promissora, bastante sensível na avaliação da depleção precoce de proteoglicanos $^{(29,30)}$.
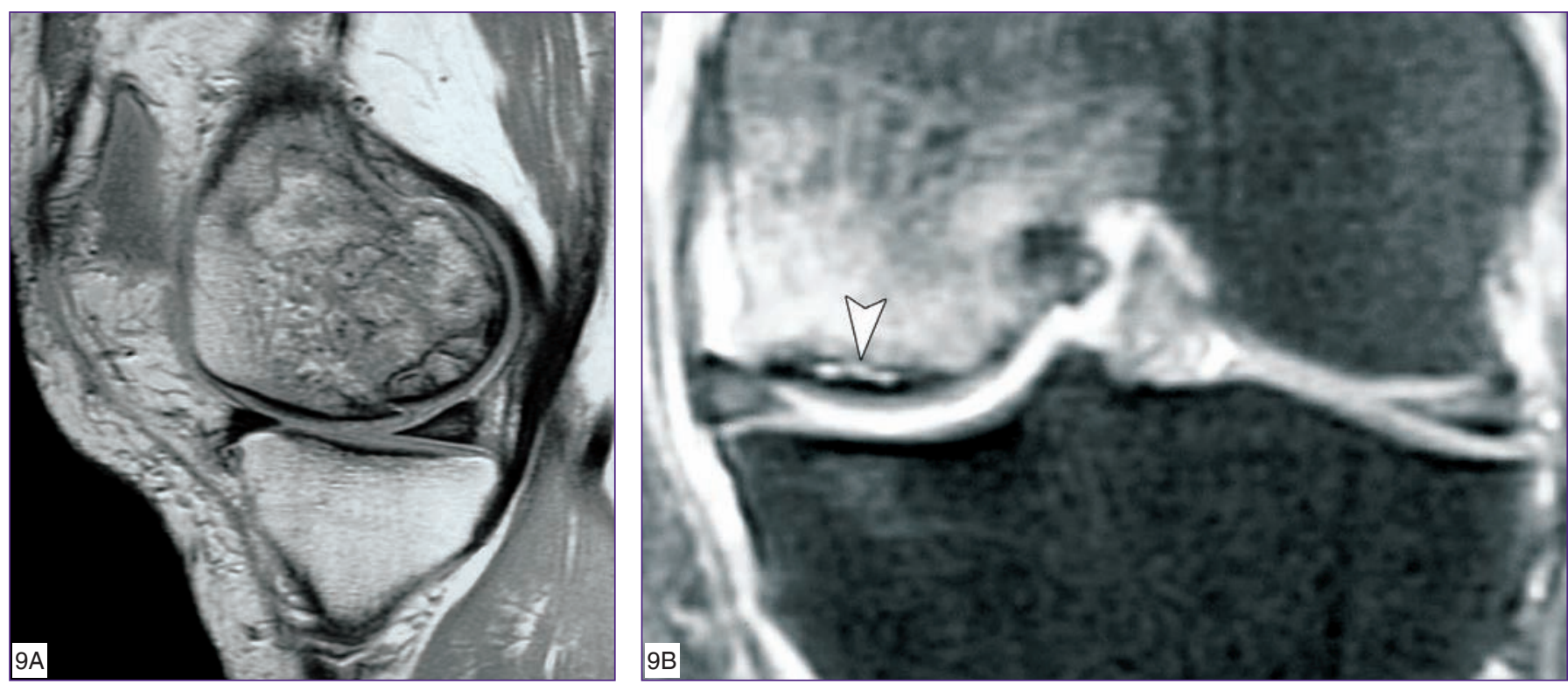

Figura 9 - Osteonecrose do côndilo femoral. (A) Imagem sagital de RM ponderada em T1 demonstra lesão geográfica no côndilo femoral medial com fratura da lâmina óssea subcondral adjacente. (B) Imagem coronal de RM ponderada em T2 com saturação de gordura evidencia artropatia degenerativa severa com necrose óssea no côndilo femoral medial associado a uma fratura da lâmina óssea subcondral adjacente (cabeça de seta).
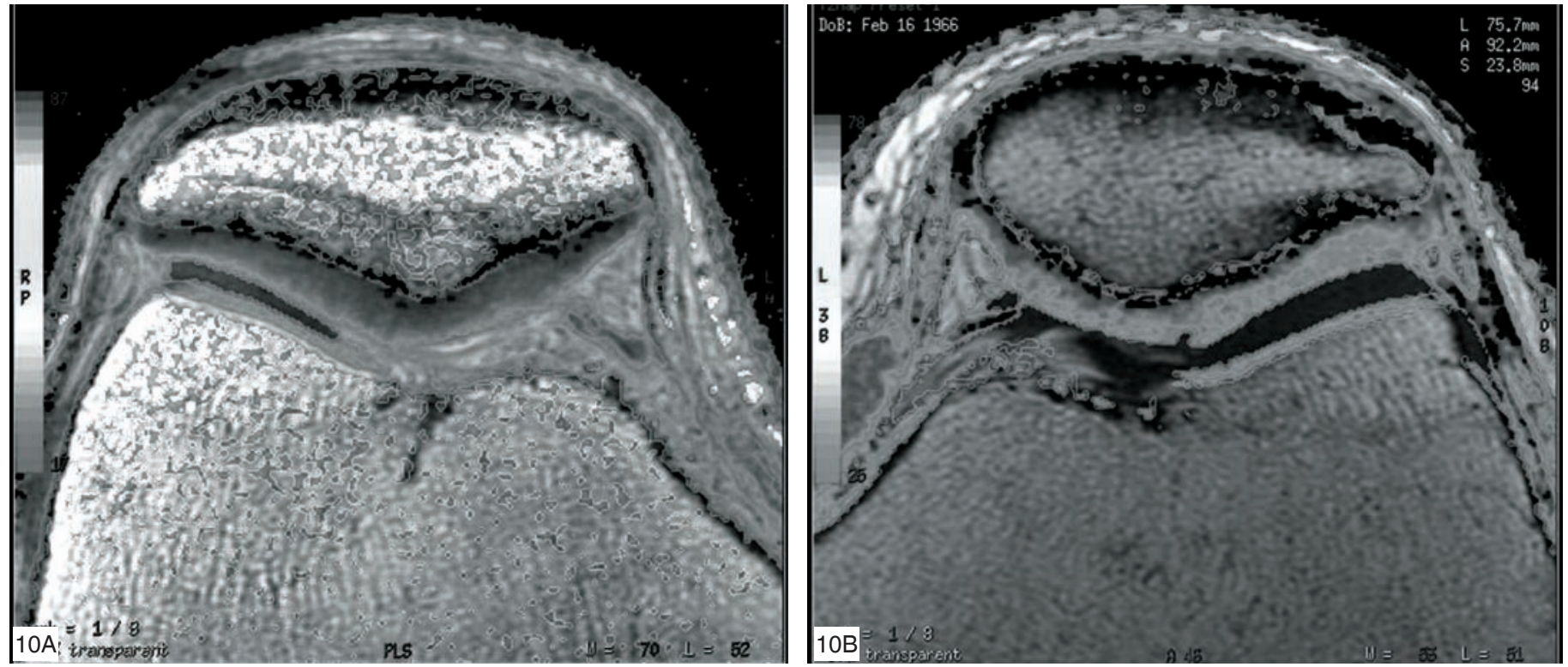

Figura 10 - Imagem funcional da cartilagem - Mapa T2. (A) Imagem axial da patela com mapa T2 representado pela escala de cores demonstra cartilagem com tempos T2 homogêneos e de valores normais (ao redor de 20mseg), indicando cartilagem normal. (B) Observa-se um aumento dos tempos T2 (ao redor de 40-50mseg), demonstrado pelo mapa de cores (setas), distribuído de forma heterogênea e mais acentuadamente na faceta lateral, indicando dano estrutural na matriz condral. Também se observa pequena fissura na faceta medial (cabeça de seta).

- d-GMERIC (delayed gadolinium-enhanced MRI of cartilage): o proteoglicano apresenta cadeias de glicosaminoglicanos, com abundantes cargas positivas. O contraste paramagnético mais utilizado, o gadolínio (Gd-DTPA) também apresenta cargas negativas. Após a injeção endo- venosa do contraste paramagnético, este penetra na cartilagem e é distribuído para áreas com baixa concentração de glicosaminoglicanos ${ }^{(28,31-33)}$. Através de um mapa T1, é possível quantificar a concentração de Gd-DTPA e, consequentemente, do conteúdo de glicosaminoglicanos. 


\section{REFERÊNCIAS}

1. Buckwalter JA, Mankin HJ. Articular cartilage: tissue design and chondrocytematrix interactions. Instr Course Lect. 1998;47:477-86.

2. Recht MP, Resnick D. Magnetic resonance imaging of articular cartilage: an overview. Top Magn Reson Imaging. 1998;9(6):328-36.

3. Recht M, Bobic V, Burstein D, Disler D, Gold G, Gray M, et al. Magnetic resonance imaging of articular cartilage. Clin Orthop Relat Res. 2001;(391 Suppl):S379-96.

4. Hodler J, Resnick D. Current status of imaging of articular cartilage. Skeletal Radiol. 1996; 25(8):703-9.

5. Disler DG, McCauley TR. Clinical magnetic resonance imaging of articular cartilage. Top Magn Reson Imaging. 1998;9(6):360-76.

6. Rubenstein JD, Li JG, Majumdar S, Henkelman RM. Image resolution and signal-to-noise ratio requirements for MR imaging of degenerative cartilage. AJR Am J Roentgenol. 1997;169(4):1089-96.

7. Schaefer FK, Kurz B, Schaefer PJ, Fuerst M, Hedderich J, Graessner J, et al. Accuracy and precision in the detection of articular cartilage lesions using magnetic resonance imaging at 1.5 Tesla in an in vitro study with orthopedic and histopathologic correlation. Acta Radiol. 2007;48(10):1131-7.

8. Mohr A. The value of water-excitation $3 D$ FLASH and fat-saturated PDW TSE MR imaging for detecting and grading articular cartilage lesions of the knee. Skeletal Radiol. 2003;32(7):396-402.

9. Potter HG, Linklater JM, Allen AA, Hannafin JA, Haas SB. Magnetic resonance imaging of articular cartilage in the knee. An evaluation with use of fast-spin-echo imaging. J Bone Joint Surg Am. 1998;80(9):1276-84.

10. Cicuttini F, Forbes A, Asbeutah A, Morris K, Stuckey S. Comparison and reproducibility of fast and conventional spoiled gradient-echo magnetic resonance sequences in the determination of knee cartilage volume. J Orthop Res. 2000;18(4):580-4.

11. Eckstein F, Schnier M, Haubner M, Priebsch J, Glaser C, Englmeier KH, et al. Accuracy of cartilage volume and thickness measurements with magnetic resonance imaging. Clin Orthop Relat Res. 1998;(352):137-48.

12. Eckstein F, Winzheimer M, Westhoff J, Schnier M, Haubner M, Englmeier $\mathrm{KH}$, et al. Quantitative relationships of normal cartilage volumes of the human knee joint--assessment by magnetic resonance imaging. Anat Embryol (Berl). 1998;197(5):383-90.

13. Eckstein F, Westhoff J, Sittek H, Maag KP, Haubner M, Faber S, et al. In vivo reproducibility of three-dimensional cartilage volume and thickness measurements with MR imaging. AJR Am J Roentgenol. 1998;170(3):593-7.

14. Yoshioka H, Stevens K, Hargreaves BA, Steines D, Genovese M, Dillingham MF, et al. Magnetic resonance imaging of articular cartilage of the knee: comparison between fat-suppressed three-dimensional SPGR imaging, fat-suppressed FSE imaging, and fat-suppressed three-dimensional DEFT imaging, and correlation with arthroscopy. J Magn Reson Imaging. 2004;20(5):857-64.

15. Kornaat PR, Reeder SB, Koo S, Brittain JH, Yu H, Andriacchi TP, et al. MR imaging of articular cartilage at 1.5T and 3.0T: comparison of SPGR and SSFP sequences. Osteoarthritis Cartilage. 2005;13(4):338-44.

16. Gold GE, Hargreaves BA, Stevens KJ, Beaulieu CF. Advanced magnetic resonance imaging of articular cartilage. Orthop Clin North Am. 2006;37(3):331-47.
17. Azer NM, Winalski CS, Minas T. MR imaging for surgical planning and postoperative assessment in early osteoarthritis. Radiol Clin North Am. 2004;42(1):43-60.

18. Vande Berg BC, Lecouvet FE, Poilvache P, Jamart J, Materne R, Lengele B, et al. Assessment of knee cartilage in cadavers with dual-detector spiral CT arthrography and MR imaging. Radiology. 2002;222(2):430-6.

19. Vande Berg BC, Lecouvet FE, Maldague B, Malghem J. MR appearance of cartilage defects of the knee: preliminary results of a spiral CT arthrographyguided analysis. Eur Radiol. 2004;14(2):208-14.

20. Vande Berg BC, Lecouvet FE, Malghem J. Frequency and topography of lesions of the femoro-tibial cartilage at spiral CT arthrography of the knee: a study in patients with normal knee radiographs and without history of trauma. Skeletal Radiol. 2002;31(11):643-9.

21. Bachmann G, Heinrichs C, Jurgensen I, Rominger M, Scheiter A, Rau WS. [Comparison of different MRT techniques in the diagnosis of degenerative cartilage diseases. In vitro study of 50 joint specimens of the knee at T1.5]. Rofo. 1997;166(5):429-36.

22. Outerbridge RE. The etiology of chondromalacia patellae. J Bone Joint Surg Br. 1961; 43:752-757.

23. Shahriaree H. Chondromalacia. Contemp Orthop. 1985;11(1):27-39.

24. Zanetti M, Bruder E, Romero J, Hodler J. Bone marrow edema pattern in osteoarthritic knees: correlation between MR imaging and histologic findings. Radiology. 2000;215(3):835-40.

25. Carrino JA, Blum J, Parellada JA, Schweitzer ME, Morrison WB. MRI of bone marrow edema-like signal in the pathogenesis of subchondral cysts. Osteoarthritis Cartilage. 2006;14(10):1081-5.

26. Poon CS, Henkelman RM. Practical T2 quantitation for clinical applications. J Magn Reson Imaging 1992;2(5):541-53.

27. Goodwin DW, Zhu H, Dunn JF. In vitro MR imaging of hyaline cartilage: correlation with scanning electron microscopy. AJR Am J Roentgenol. 2000;174(2):405-9.

28. Burstein D, Bashir A, Gray ML. MRI techniques in early stages of cartilage disease. Invest Radiol. 2000;35(10):622-38.

29. Duvvuri U, Charagundla SR, Kudchodkar SB, Kaufman JH, Kneeland JB, Rizi $\mathrm{R}$, et al. Human knee: in vivo T1(rho)-weighted MR imaging at 1.5 T--preliminary experience. Radiology. 2001;220(3):822-6.

30. Regatte RR, Akella SV, Borthakur A, Kneeland JB, Reddy R. In vivo proton MR three-dimensional T1rho mapping of human articular cartilage: initial experience. Radiology. 2003;229(1):269-74.

31. Tiderius CJ, Tjornstrand J, Akeson P, Sodersten K, Dahlberg L, Leander P. Delayed gadolinium-enhanced MRI of cartilage (dGEMRIC): intra- and interobserver variability in standardized drawing of regions of interest. Acta Radiol. 2004;45(6):628-34.

32. Tiderius CJ, Svensson J, Leander P, Ola T, Dahlberg L. dGEMRIC (delayed gadolinium-enhanced MRI of cartilage) indicates adaptive capacity of human knee cartilage. Magn Reson Med. 2004;51(2):286-90.

33. Kim YJ, Jaramillo D, Millis MB, Gray ML, Burstein D. Assessment of early osteoarthritis in hip dysplasia with delayed gadolinium-enhanced magnetic resonance imaging of cartilage. J Bone Joint Surg Am. 2003;85(10):1987-92. 